\title{
DERIVED FUNCTORS AS HOMOTOPY GROUPS
}

\author{
A. K. MODAWI
}

(Received 26 January 1989)

Communicated by J. H. Rubinstein

\begin{abstract}
In this paper a generalization of the interpretation of Robinson of torsion products as homotopy groups is given. This generalization allows us to define right derived functors of a coproduct preserving functor defined on a small category with all finite colimits to the category of abelian groups. We show that when the category is additive the definition coincides with the definitions of the right derived functors of Cartan and Eilenberg.
\end{abstract}

1980 Mathematics subject classification (Amer. Math. Soc.) (1985 Revision): 18 E 25, 18 G 10. Keywords and phrases: Categories, derived functors, infinite loop spaces, spectra.

\section{Introduction}

In his paper [5] A. C. Robinson interpreted the torsion products $\operatorname{Tor}_{s}^{\Lambda}(M, N)$, where $M$ and $N$ are respectively right and left modules over an associative ring $\Lambda$ with an identity, as the homotopy groups of a certain category $T^{\Lambda}(M, N)$.

We will modify the definition of $T^{\Lambda}(M, N)$ so that it is general enough to allow us to associate to any coproduct preserving covariant functor $T: \xi \rightarrow$ $\mathbf{A b}$, a sequence of coproduct preserving functors $T_{i}: \xi \rightarrow \mathbf{A b}$; where $\xi$ is a category with small hom sets, which is finite complete, finite cocomplete, and has a null object $*$. It is also assumed that $\xi$ has enough projective objects. As usual, Ab is the category of abelian groups. If $\xi$ is an additive category and $T$ an additive functor the sequence $\left[T_{i}\right]_{i \geq 0}$ coincides with the sequence of the right derived functors of $T$, whose axioms are given in [3].

(C) 1990 Australian Mathematical Society $0263-6115 / 90 \$ A 2.00+0.00$ 
Throughout this paper categorical notations and terminologies conform to those of [4].

To outline our result we will define a category $T^{\xi}(M)$ (the analogue of $\left.T^{\Lambda}(M, N)\right)$ for each object $M$ of $\xi$ as follows.

An object of $T^{\xi}(M)$ is a triple $(P, f, x)$, where $P$ is a projective object of $\xi, f: P \rightarrow M$ is an $\xi$-morphism and $x$ is an element of $T(P)$. A morphism of $T^{\xi}(M),\left(P_{1}, f_{1}, x_{1}\right) \rightarrow\left(P_{2}, f_{2}, x_{2}\right)$ consists of an $\xi$-morphism $P_{1} \stackrel{\phi}{\longrightarrow} P_{2}$ such that $f_{2} \circ \phi=f_{1}$ and $T(\phi)\left(x_{1}\right)=x_{2}$.

If we assume that the collection of all projective objects of $\xi$ forms a set then $T^{\xi}(M)$ becomes a small category. The coproduct on $\xi$, together with the fact that $T$ preserves coproducts (up to natural isomorphisms) gives a bifunctor $\boxplus: T^{\xi}(M) \times T^{\xi}(M) \rightarrow T^{\xi}(M)$ given by $\left(P_{1}, f_{1}, x_{1}\right) \boxplus\left(P_{2}, f_{2}, x_{2}\right)=$ $\left(P_{1} \amalg P_{2}, f_{1} \amalg f_{2}, x_{1} \oplus x_{2}\right)$. Note that $\amalg$ is used for the coproduct on $\xi$, and $\oplus$ is used for the coproduct on $\mathbf{A b}$.

The bifunctor $\boxplus$ gives rise to a spectrum whose first term is the nerve $\left|T^{\xi}(M)\right|$ of the category $T^{\xi}(M)$. One obtains this spectrum by employing Segal's machine for constructing infinite loop spaces [6]. Our main theorems, which generalise [5, Theorems 1 and 2], are the following theorems.

THEOREM 1. (i) The bifunctor $\boxplus$ induces a structure of an abelian group on $\pi_{0}\left|T^{\xi}(m)\right|$. Therefore the spectrum constructed from $\left(T^{\xi}(M), \boxplus\right)$ is an

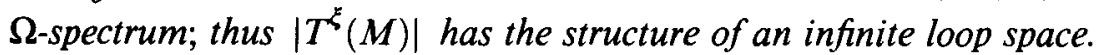

(ii) There is a natural homomorphism $\pi_{0}\left|T^{\xi}(M)\right| \rightarrow T(M)$ which is an isomorphism if $T$ is right-exact. (For the definition of right-exactness see Note 1 below.)

(iii) If $\xi$ is an additive category, then there are natural isomorphisms

$$
\pi_{s}\left|T^{\xi}(M)\right| \approx T_{s}(M), \quad s \geq 0,
$$

where $\left[T_{s}\right]_{s \geq 0}$ are the right derived functors of $T$.

Note 1. A functor $S: \boldsymbol{\xi} \rightarrow \mathbf{A b}$ is said to be right-exact if it preserves epimorphisms and has the Mayer-Vietoris property, that is, for every diagram $A \underset{g}{\stackrel{f}{\rightrightarrows}} B$ in $\xi$ and $x \in S(A)$ such that $S(f)(x)=S(g)(x)$, there exists a morphism $C \stackrel{h}{\longrightarrow} A$ and an element $y \in S(C)$ such that $S(h)(y)=x$.

For our next theorem, which generalises [5, Theorem 2], we will assume, further to the previous assumptions on $\xi$, the following conditions. 
(i) There is a bifunctor $\square: \xi \times \xi \rightarrow \xi$ which makes $\xi$ a monoidal category, in which the functors $R \square-: \xi \rightarrow \xi$, and $-\square R: \xi \rightarrow \xi$ commute (up to natural isomorphisms) with the coproduct on $\xi$, for every object $R$ of $\xi$.

(ii) For every pair of objects $M$ and $N$ of $\xi, T(M \square N)$ is naturally isomorphic to $T(M) \otimes T(N)$.

(iii) The unit object $\Omega$ of $\square$ is a projective object of $\xi$ and $P_{1} \square P_{2}$ is projective whenever $P_{1}$ and $P_{2}$ are projective.

Now under these assumptions we have the following theorem.

THEOREM 2. As an infinite loop space, $\left|T^{\xi}(M)\right|$ has the homotopy type of a product of Eilenberg-Mac Lane spaces.

\section{The proof of Theorem 1}

(i) The bifunctor $\boxplus$ gives $\pi_{0}\left|T^{\xi}(M)\right|$ an abelian monoid structure in the obvious way. To complete the proof of (i) it is enough to show that every member of $\pi_{0}\left|T^{\xi}(M)\right|$ has an additive inverse. So let $\alpha \in \pi_{0}\left|T^{\xi}(M)\right|$ be represented by $(P, f, x)$. Let $\beta$ be the element represented by $(P, f,-x)$; then $\alpha+\beta$ is represented by $(P \amalg P, f \amalg f, x \oplus-x)$, by the bifunctor $\boxplus$. Now $T(f \amalg f)(x \oplus-x)=x+(-x)=0 \in T(M)$. ( $T$ is coproduct preserving.) Thus there are morphisms $(P \amalg P, f \amalg f, x \oplus-x) \stackrel{\text { id山id }}{\longrightarrow}$ $(P, f, 0) \leftarrow(*, *, 0)$ where * denotes the null object as well as the null morphism $* \rightarrow M$. It follows that $\alpha+\beta=0$.

We also conclude that the spectrum constructed from $\left(T^{\xi}(M), \amalg\right)$ is an $\Omega$-spectrum in which the first term $\left|T^{\xi}(M)\right|$ is an infinite loop space.

(ii) The map $\pi_{0}\left|T^{\xi}(M)\right| \rightarrow T(M)$ which sends $(P, f, x)$ to $T(f)(x)$ is easily seen to be a natural homomorphism.

Suppose now that $T$ is right-exact (in the sense of Note 1). Since $\xi$ has enough projectives there is a projective object $P$ and an $\xi$-epimorphism $P \stackrel{f}{\longrightarrow} M$. Now $T(P) \stackrel{T(f)}{\longrightarrow} T(M)$ is an epimorphism in $\mathbf{A b}$. This shows that the natural homomorphism $\pi_{0}\left|T^{\xi}(M)\right| \rightarrow T(M)$ is an epimorphism. To show that $\pi_{0}\left|T^{\xi}(M)\right| \rightarrow T(M)$ is one-to-one, suppose that $\left(P_{1}, f_{1}, x_{1}\right)$ and $\left(P_{2}, f_{2}, x_{2}\right)$ are such that $T\left(f_{1}\right)\left(x_{1}\right)=T\left(f_{2}\right)\left(x_{2}\right)$. Then clearly $\left(P_{1} \amalg P_{2}\right.$, $\left.f_{1} \amalg *, x_{1} \oplus x_{2}\right)$ and $\left(P_{1} \amalg P_{2}, * \amalg f_{2}, x_{1} \oplus x_{2}\right)$ map to the same element in $T(M)$. By right-exactness of $T$ there is an object $D$ of $\xi$, a morphism $g: D \rightarrow P_{1} \amalg P_{2}$ and $x \in T(D)$ such that $\left(* \amalg f_{2}\right) \circ g=\left(f_{1} \amalg *\right) \circ g$ and 
$T(g)(x)=x_{1} \oplus x_{2}$. Now there is a projective object $P$ in $\xi$, a morphism $k: P \rightarrow D$ and an element $y \in T(P)$ such that $T(k)(y)=x$. It is now straightforward to see that the $\xi$-morphism $P \stackrel{k}{\longrightarrow} D \stackrel{g}{\longrightarrow} P_{1} \amalg P_{2}$ gives rise to a pair of $T^{\xi}(M)$-morphisms from $(P,(* \amalg f) \circ g \circ k, y)$ to the objects $\left(P_{1} \amalg P_{2}, f_{1} \amalg *, x_{1} \oplus x_{2}\right)$ and $\left(P_{1} \amalg P_{2}, * \amalg f_{2}, x_{1} \oplus x_{2}\right)$. These morphisms together with the obvious morphisms $\left(P_{1}, f_{1}, x_{1}\right) \rightarrow\left(P_{1} \amalg P_{2}, f_{1} \amalg *, x_{1} \oplus\right.$ $\left.x_{2}\right)$ and $\left(P_{2}, f_{2}, x_{2}\right) \rightarrow\left(P_{1} \amalg P_{2}, * \amalg f_{2}, x_{1} \oplus x_{2}\right)$ imply that $\left(P_{1}, f_{1}, x_{1}\right)$ and $\left(P_{2}, f_{2}, x_{2}\right)$ represent the same element in $\pi_{0}\left|T^{\xi}(M)\right|$.

(iii) The proof of this part is similar to the proof of [5, Theorem 1(ii)].

\section{The proof of Theorem 2}

First recall the description of the $\Gamma$-space structure $\left|T^{\xi}(M)\right|$ as given in [5, Section 2] For each object $S^{+}$of $\Gamma$, introduce the category $T^{\xi}(M)_{S^{+}}$. An object of $T^{\xi}(M)_{S^{+}}$is a triple $(P, f, \mathbf{x})$ where $P$ is a sheaf of projective objects on the canonical topology $J_{S^{+}}$on the category of all based subsets of $S^{+}, f: P \rightarrow M$ is a homomorphism to the constant sheaf defined by $M$, and $\mathbf{x}: J_{S^{+}} \rightarrow T(P)$ is a sheaf homomorphism into the sheaf $T(P)$. A morphism $\left(P_{1}, f_{1}, \mathbf{x}_{1}\right) \rightarrow\left(P_{2}, f_{2}, \mathbf{x}_{2}\right)$ is specified by a sheaf homomorphism $g: P_{1} \rightarrow P_{2}$ such that $T(g) \circ \mathbf{x}_{1}=\mathbf{x}_{2}$. Any $\Gamma$-morphism $S^{+} \rightarrow R^{+}$yields a morphism of topologies and hence a functor $T^{\xi}(M)_{S^{+}} \rightarrow T^{\xi}(M)_{R^{+}}$. Passing to the nerve we have a $\Gamma$-space $S^{+} \rightarrow\left|T^{\xi}(M)_{S^{+}}\right|$which is readily seen to be special [5, A.1].

Now for the category $\xi$ and the functor $T: \xi \rightarrow$ Ab satisfying the conditions in Theorem 2 , there is a pairing of $\Gamma$-spaces $T^{\xi}(M) \times T^{\xi}(N) \rightarrow$ $T^{\xi}(M \square N)$, (in the sense of $[5$, Section $\left.5, A]\right)$. The $\Gamma \times \Gamma$-space needed to set up the pairings is the nerve of the category $T^{\xi}(M: N)$ (the analogue of $T^{\left(\Lambda: \Lambda^{\prime}\right)}(M, N ; M, N)$ of [5]). An object of this category is a quadruple $\left(X_{S^{+}}, Y_{R^{+}} ; Z_{S^{+} \wedge R^{+}}, m\right)$ where $X_{S^{+}}, Y_{R^{+}}, Z_{S^{+} \wedge R^{+}}$are objects of $T^{\xi}(M)_{S^{+}}, T^{\xi}(N)_{R^{+}}$and $T^{\xi}(M \square N)_{S^{+} \wedge R^{+}}$respectively.

(Note that $T(M \square N)_{S^{+} \wedge R^{+}}$represents the $\Gamma \times \Gamma$-space $\left.\left(S^{+}, R^{+}\right) \rightarrow\left|T^{\xi}(M \square N)_{S^{+} \wedge R^{+}}\right|.\right)$ The map $m: X_{S^{+}} \times Y_{R^{+}} \rightarrow Z_{S^{+} \wedge R^{+}}$is a map of sheaves that arises from the functor $\hat{\square}: T^{\xi}(M) \times T^{\xi}(N) \rightarrow T^{\xi}(M \square N)$ given by

$$
\left(\left(P_{1}, f_{1}, x_{1}\right) ;\left(P_{2}, f_{2}, x_{2}\right)\right) \rightarrow\left(P_{1} \square P_{2}, f_{1} \square f_{2}, x_{1} \otimes x_{2}\right) ;
$$

one easily sees that $\hat{\square}$ respects $\boxplus$-products in both variables. We also assume that $m$ respects the augmentation to the appropriate constant sheaves. 
The structural morphisms needed to define the pairings are given by two functors. To obtain the first functor we consider the functor $\left(X_{S^{+}}, Y_{R^{+}}\right.$; $\left.Z_{S^{+} \wedge R^{+}}, m\right) \rightarrow Z_{S^{+} \wedge R^{+}}$which induces on nerves the $\Gamma \times \Gamma$-map $\mu\left(S^{+}, R^{+}\right)$:

$$
\left|T^{\xi}(M: N)_{\left(S^{+}, R^{+}\right)}\right| \rightarrow\left|T^{\xi}(M \square N)_{S^{+} \wedge R^{+}}\right| .
$$

Next we consider the functor $\left(X_{S^{+}}, Y_{R^{+}} ; Z_{S^{+} \wedge R^{+}}, m\right) \rightarrow\left(X_{S^{+}}, Y_{R^{+}}\right)$which can be seen to be an equivalence of the categories $T^{\xi}(M: N)$ and $T^{\xi}(M) \times$ $T^{\xi}(N)$ and so induces a weak homotopy equivalence $k\left(S^{+}, R^{+}\right)$on the nerves. We therefore deduce from [5, A.11] the existence of an associated pairing of spectra $S_{p} T^{\xi}(M) \wedge S_{p} T^{\xi}(N) \rightarrow S_{p} T^{\xi}(M \square N)$. If we set $N=\Omega$, the unit of $\square$ the pairing becomes

$$
S_{p} T^{\xi}(M) \wedge S_{p} T^{\xi}(\Omega) \rightarrow S_{p} T^{\xi}(M)
$$

Since $\Omega$ is a projective object in $\xi, S_{p} T^{\xi}(\Omega)$ is an Eilenberg-Mac Lane spectrum. Now we have a straightforward generalisation of $[5$, Theorem 2.5].

THEOREM 3. The pairing makes the spectrum $S_{p} T^{\xi}(M)$ and $S_{p} T^{\xi}(\Omega)$ module with respect to the map $\Sigma \rightarrow S_{p} T^{\xi}(M)$ which represents $1 \in \pi_{0}\left|T^{\xi}(M)\right|$; $\Sigma$ being the sphere spectrum.

Proof. See the proof of [5, Theorem 2.5].

To complete the proof of Theorem 2, we observe that an argument of Moore and Whitehead [7] shows that $S_{p} T^{\xi}(M)$ has the homotopy type of a product of an Eilenberg-Mac Lane spectra.

\section{References}

[1] M. Andre, Méthode simpliciale en algèbre homologique et algèbre commutative, (Lecture Notes in Math. 32, Springer-Verlag, Berlin, Heidelberg and New York, 1967).

[2] M. Artin, Grothendieck topologies (Harvard, 1962).

[3] H. Cartan and S. Eilenberg, Homological algebra (Princeton, 1956).

[4] S. Mac Lane, Categories for the working mathematician (Springer-Verlag, New York, Heidelberg and Berlin, 1971).

[5] A. C. Robinson, 'Torsion products as homotopy groups', J. Pure Appl. Algebra 21 (1981), 167-182. 
[6] G. B. Segal, 'Categories and cohomology theories', Topology 13 (1974), 293-312.

[7] G. W. Whitehead, 'Generalised homology theories', Trans. Amer. Math. Soc. 102 (1962), 227-283.

Department of Mathematics and Computer Sciences

U.A.E. University

P. O. Box 15551

Al-Ain

United Arab Emirates 